\title{
Vorwort
}

Als Nachschlagewerk für das gesamte öffentliche Leben im Freistaat Bayern erscheint das Bayerische Jahrbuch in seiner 98. Ausgabe vollständig überarbeitet und aktualisiert. Die verzeichneten Institutionen haben durch großes Interesse und mit viel Hilfsbereitschaft unsere Recherchen unterstützt und eine gute und enge Zusammenarbeit ermöglicht.

Wie gewohnt bietet das Bayerische Jahrbuch 2019 vielfältige Informationen über Aufbau und Aufgaben von Behörden, Ministerien und Körperschaften und der mit innen verbundenen Einrichtungen. Alle Angaben zur staatlichen und kommunalen Verwaltung Bayerns, wie auch zu den Kirchenbehörden und Religionsgemeinschaften, zu Verbänden sowie zu den öffentlich-rechtlichen Kreditinstituten wurden aktualisiert und ergänzt.

Die vorliegende Ausgabe dokumentiert die Ergebnisse der Bezirkstags- und der Landtagswahl 2018 und berücksichtigt daraus resultierende wichtige Neuerungen wie die Umbenennung einzelner Ministerien aufgrund der Änderung der Geschäftsbereiche im neuen bayerischen Kabinett. Wir müssen jedoch darauf hinweisen, dass bei Redaktionsschluss keine genaueren Angaben zum Geschäftsverteilungsplan einzelner bayerischer Ministerien vorlagen.

Das Bayerische Jahrbuch 2019 beginnt mit den Kapiteln über den Bayerischen Landtag, die bayerischen Staatsbehörden und die bayerischen Staatsministerien. Sie zeigen Aufbau und Hierarchieebenen innerhalb der einzelnen Ministerien und führen die Einträge der angeschlossenen bzw. untergeordneten Institutionen auf.
Zwei Kapitel zur Bundesrepublik Deutschland (Bundestag, Bundesrat, Bundesregierung etc.) sowie zu den Behörden und Dienststellen der Bundesrepublik Deutschland in Bayern schließen den staatlichen Teil des Jahrbuchs ab.

Es folgen die Kapitel „Kommunale Verwaltung“ mit den Einträgen der bayerischen Gemeinden, Städte, Verwaltungsgemeinschaften, Landkreise und Bezirke, „Religionsgemeinschaften“, „Vereinigungen, Verbände und Organisationen“ sowie „Öffentlich-rechtliche Kreditinstitute".

Der Hauptteil des Jahrbuches wird durch einen Statistischen Überblick, der in einem Tabellenteil ausgewählte Daten zu Bayern beinhaltet, abgeschlossen.

Der Registerteil des Buches umfasst ein umfangreiches Personenregister mit ca. 12850 verzeichneten Personen sowie ein Sach- und Institutionenregister mit mehr als 6600 im Buch aufgeführten Einrichtungen, die eine gezielte Suche im Bayerischen Jahrbuch 2019 ermöglichen.

Marlies Janson
Redaktion Bayerisches Jahrbuch

München, im November 2018 


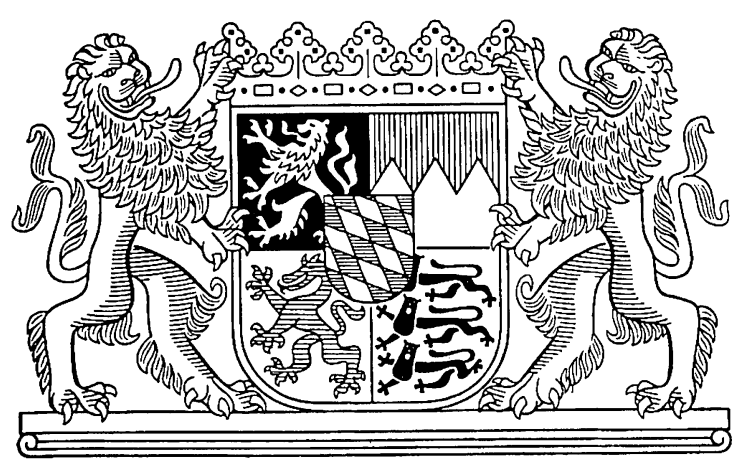

\section{Das Große Bayerische Staatswappen}

Bayerns Staatswappen wurde am 5. Juni 1950 mit dem „Gesetz über das Wappen des Freistaates Bayern" eingeführt. Die einzelnen heraldischen Teile haben folgenden historischen Hintergrund:

Der goldene Löwe im schwarzen Feld war ursprünglich das Symbol der Pfalzgrafen bei Rhein und diente nach der Belehnung des bayerischen Herzogs Ludwig im Jahre 1214 mit der Pfalzgrafschaft jahrhundertelang als gemeinsames Kennzeichen der altbayerischen und pfälzischen Wittelsbacher. Heute steht der Pfälzer Löwe für den Regierungsbezirk Oberpfalz.

Der fränkische Rechen erschien zuerst um 1350 als Wappen einiger Orte des Hochstifts Würzburg und seit 1410 auch in den Siegeln der Fürstbischöfe. Die drei weißen (silbernen) Spitzen im roten Feld symbolisieren heute die drei fränkischen Regierungsbezirke Oberfranken, Mittelfranken und Unterfranken.

Der blaue Panther im weißen (silbernen) Wappenfeld wurde von den in Niederbayern ansässigen Pfalzgrafen von Ortenburg geführt (12. Jahrhundert) und später von den Wittelsbachern übernommen. Heute steht dieses Symbol für die altbayerischen Regierungsbezirke Niederbayern und Oberbayern.

Die drei übereinanderschreitenden schwarzen Löwen sind das alte Wappen der Hohenstaufen (erstmals 1216), der einstigen Herzöge von Schwaben. Sie repräsentieren im Staatswappen den Regierungsbezirk Schwaben.

Die weißblauen Rauten sind das bayerische Wahrzeichen schlechthin. Sie waren seit 1204 im Wappen des Grafen von Bogen zu finden. 1247 übernahmen die Wittelsbacher die Rauten als Stammwappen. Die Anordnung beginnt traditionell mit einer weißen Raute in der heraldisch rechten oberen Schildecke. Darauf beruht die Farbenfolge Weiß und Blau in der Landesflagge. Heute repräsentiert der Rautenschild Bayern als Ganzes. Er dient als ,Kleines Staatswappen".

Die Volkskrone, erstmals im Wappen von 1923, bezeichnet nach dem Wegfall der Königskrone die Volkssouveränität. Die beiden schildhaltenden Löwen setzen eine Tradition aus dem 14. Jahrhundert fort. 
Bayerisches Staatsministerium des Innern, für Sport und Integration

Bayerisches Staatsministerium für Wohnen, Bau und Verkehr

37

Bayerisches Staatsministerium der Justiz

Bayerisches Staatsministerium für Unterricht und Kultus

59

Bayerisches Staatsministerium für Wissenschaft und Kunst

Bayerisches Staatsministerium der Finanzen und für Heimat

91

Bayerisches Staatsministerium für Wirtschaft, Landesentwicklung und Energie

Bayerisches Staatsministerium für Umwelt und Verbraucherschutz

Bayerisches Staatsministerium für Ernährung, Landwirtschaft und Forsten

Bayerisches Staatsministerium für Familie, Arbeit und Soziales

137

Bayerisches Staatsministerium für Gesundheit und Pflege

143

Bayerisches Staatsministerium für Digitales

153

Bundesrepublik Deutschland

154

Behörden und Dienststellen der Bundesrepublik Deutschland in Bayern

177

Kommunale Verwaltung Bayerns

189

\begin{tabular}{lcc}
\hline & Religionsgemeinschaften & 364 \\
\hline & Vereinigungen, Verbände und Organisationen & 377 \\
\hline & Öffentlich-rechtliche Kreditinstitute & 461 \\
\hline Statistischer Überblick & 465 \\
\hline & Abkürzungsverzeichnis & 508 \\
\hline
\end{tabular}

